\title{
Method development for detecting the novel cyanide antidote dimethyl trisulfide from blood and brain, and its interaction with blood
}

Lorand Kiss ${ }^{1}$, Secondra Holmes ${ }^{1}$, Ching-En Chou ${ }^{1}$, Xinmei Dong ${ }^{1}$, James Ross ${ }^{1}$, Denise Brown $^{1}$, Brooke Mendenhall ${ }^{1}$, Valerie Coronado ${ }^{1}$, Deepthika De Silva ${ }^{1}$, Gary A. Rockwood ${ }^{2}$, Ilona Petrikovics ${ }^{1}$, David E. Thompson ${ }^{1 *}$

${ }^{1}$ Department of Chemistry, Sam Houston State University, Huntsville, Texas, 77341, USA

${ }^{2}$ U.S. Army Medical Research Institute of Chemical Defense, 2900 Rickets Point Road, Aberdeen Proving Ground, MD, 21010, USA

*Corresponding author: David E. Thompson, Associate Professor of Chemistry, Sam Houston State University, P.O. Box: 2117, Huntsville, TX USA; Tel/Fax: 1-936-294-3270, 1936-294-4996; E-mail: david.thompson@shsu.edu

\begin{abstract}
The antidotal potency of dimethyl trisulfide (DMTS) against cyanide poisoning was discovered and investigated in our previous studies. Based on our results it has better efficacy than the Cyanokit and the Nithiodote therapies that are presently used against cyanide intoxication in the US. Because of their absence in the literature, the goal of this work was to develop analytical methods for determining DMTS from blood and brain that could be employed in future pharmacokinetic studies. An HPLC-UV method for detection of DMTS from blood, a GC-MS method for detection of DMTS from brain, and associated validation experiments are described here. These analytical methods were developed using in vitro spiking of brain and blood, and are suitable for determining the in vivo DMTS concentrations in blood and brain in future pharmacokinetic and distribution studies. An important phenomenon was observed in the process of developing these methods. Specifically, recoveries from fresh blood spiked with DMTS were found to be significantly lower than recoveries from aged blood spiked in the same manner with DMTS. This decreased DMTS recovery from fresh blood is important, both because of the role it may play in the antidotal action of DMTS in the presence of cyanide, and because it adds the requirement of sample stabilization to the method development process. Mitigation procedures for stabilizing DMTS samples in blood are reported.
\end{abstract}




\section{Keywords}

dimethyl trisulfide, dimethyl disulfide, dibuthyl disulfide, HPLC, GC-MS, SPME

\section{Abbreviations}

ACN, acetonitrile; DBDS, dibuthyl disulfide; DMDS, dimethyl disulfide; DMTS, dimethyl trisulfide; $\mathrm{EtOH}$, ethanol; $\mathrm{MeOH}$, methanol; PDMS, polydimethyl siloxane; SPME, solid phase micro extraction 


\section{Introduction}

Polysulfides (mainly di- and trisulfides) are molecules that are naturally found in vegetables such as garlic, asparagus, and cabbage. Beyond their characteristic odor they often exhibit beneficial health effects related primarily to cardiovascular protection [1]. Dimethyl trisulfide (DMTS) is a characteristic component of the smells associated with the vegetables mentioned above, cheeses [2], and metabolic products of bacteria. DMTS is thus used in the food industry as a flavor enhancer [3]. DMTS is also one the odorant molecules that emanate from decomposing animals [4]. Recently the antifungal [5] and larvicidal [6] activities of DMTS have been reported. DMTS has also been shown to have promising properties as a cyanide antidote [7-9].

Cyanide is an acute toxic species that interferes with aerobic respiration. By binding to the heme iron in the mitochondrial cytochrome c oxidase, cyanide prevents the cells' oxygen utilization and thus shuts down ATP production [10]. This intoxication is naturally mitigated by the intracellular sulfurtransferase enzyme rhodanese, which is able to convert cyanide to the less toxic thiocyanate in the presence of a sulfur donor. However, beyond a certain dose the body is not able to convert the cyanide to thiocyanate, primarily because of the depletion of the endogenous sulfur donor stores.

One approach to treating cyanide intoxication is to boost the concentration of sulfur donors in the body. In recent in vitro experiments, DMTS has demonstrated promise as an exogenous sulfur donor for the rhodanese enzyme [9]. Furthermore, DMTS has also shown some capacity to convert cyanide to thiocyanate even in the absence of rhodanese. Building on the promise of these in vitro studies, early in vivo efficacy studies have shown superior antidotal properties of DMTS relative to the commercially available cyanide antidotes (Nithiodote $^{\mathrm{TM}}$ and Cyanokit®) [9].

While the present commercially available cyanide antidotes have been formulated for intravenous administration, there is also a need for formulations that can be delivered more rapidly by untrained personnel in mass casualty scenarios. In order to meet this challenge, an intramuscular formulation was developed for DMTS, which can be suitable for rapid selfadministration [7]. Pharmacokinetic studies are needed to characterize the rate at which DMTS is distributed from the injection site into the blood, and from blood vessels into the organs such as the brain.

To support these studies, analytical methods for determining DMTS from blood and organ tissues are needed. Developing a quantitative analytical method for DMTS detection 
from blood would be necessary to investigate the absorption of DMTS and to reveal the interaction between DMTS and cyanide in blood. The organ that is of greatest interest in the context of cyanide intoxication is the brain. A method for detecting DMTS from brain would enable characterization of the permeability of DMTS through blood-brain barrier, and determination of the rate of accumulation of the antidote in the brain.

Methods have been reported that use GC-MS [4, 11-13] for DMTS detection, and recently Manandhar et al. [14] described a validated analytical procedure for DMTS detection from blood. In parallel with the work of Manandhar et al. [14] another analytical method was created by our group for DMTS analysis in blood. However, to the best of our knowledge there is no quantitative method for the analysis of DMTS from brain. In this paper, we report the development of sample preparation and analytical methods for the detection of DMTS from blood and brain samples. A SPME-GC-MS method was developed for DMTS detection from brain, and an HPLC-UV method for DMTS detection from blood. In labs like ours that are equipped with a single GC-MS and a single HPLC-UV instrument, the development of distinct methods enables the blood and brain samples associated with a specific animal study to be analyzed in parallel, providing a more rapid and efficient analytical strategy than would be possible if both methods made use of the same instrument. Method validations followed the FDA and ICH guidelines.

\section{Materials and Methods}

\subsection{Chemicals and Equipment}

HPLC grade water, and sodium chloride were purchased from J.T. Baker (Center Valley, PA, USA). HPLC grade acetonitrile and ethanol were purchased from Acros Organics (Thermo Fisher Scientific, Geel, Belgium), and methanol from EMD Chemicals Inc. (Billerica, MA, USA). Polysorbate 80 was purchased from Alfa Aesar (Ward Hill, MA, USA). DMTS, dimethyl disulfide (DMDS), sodium heparin, and sodium hydroxide were purchased from Sigma Aldrich (SAFC, St Louis, MO, USA). Dibutyl disulfide (DBDS) was purchased from TCI America (Portland, OR, USA). $100 \mu \mathrm{L}$ Hamilton gas tight luer-lock syringes, $1.7 \mathrm{ml}$ micro centrifuge tubes, screw cap vials $(1.5 \mathrm{ml}$ and $5 \mathrm{ml}) 27 \mathrm{G} 1 / 2$ needles were purchased from VWR International (Radnor, PA, USA). Inserts $(250 \mu 1)$ with polymer feet were purchased from Agilent Technologies (Santa Clara, CA, USA). HPLC experiments employed a Dionex Ultimate 3000 (Thermo Scientific, Waltham, MA, USA) HPLC-UV instrument. 


\subsection{Method development for DMTS detection from blood}

An HPLC-UV method was developed for DMTS detection from blood. The sample preparation was initiated by spiking $495 \mu \mathrm{l}$ of heparinized and defibrinated sheep blood (Carolina, Burlington, NC, USA) with $5 \mu \mathrm{l}$ of ethanolic DMTS $(10-10000 \mu \mathrm{g} / \mathrm{ml})$ solution. Then $200 \mu 1$ of ice cold $0.1 \mathrm{mg} / \mathrm{ml}$ DMDS (internal standard) in a suitable solvent (ethanol, acetonitrile, or methanol) was mixed with an $80 \mu \mathrm{l}$ aliquot of DMTS-spiked blood in an Eppendorf tube. The Eppendorf tubes were vortexed at room temperature, and then centrifuged at $14000 \mathrm{rcf}$ at $4{ }^{\circ} \mathrm{C}$ for 5 minutes to sediment the debris and precipitated proteins.

An $80 \mu \mathrm{l}$ aliquot of the clear supernatant was transferred to HPLC vials with glass insert. From this 20,30 , or $40 \mu \mathrm{l}$ was auto sampled and injected through a guard column onto a $250 \times 4.60 \mathrm{~mm}$ non-polar C-8 analytical HPLC column having a Phenomenex Luna stationary phase consisting of bonded octane units coated on silica support particles (pore size of $100 \AA$, outer diameter of $5 \mu \mathrm{m}$ ). Blends of water and acetonitrile, in ratios between 40:60 and 30:70, were explored as the mobile phase, using a flow rate of $1 \mathrm{ml} / \mathrm{min}$. Analyte absorbance at the $215 \mathrm{~nm}$ wavelength was monitored by a UV detector.

Two control experiments were carried out to check for changes in DMTS recovery based on the age of blood and length of incubation time in plasma. In the first control experiment the blood was spiked with $50 \mu \mathrm{g}$ of DMTS per ml of blood immediately after it arrived from the supplier (fresh blood) and three days later (old blood). The DMTS concentration in the fresh and old blood was determined immediately after spiking. In the second control experiment the blood was spiked with $50 \mu \mathrm{g}$ of DMTS per ml of blood, mixed with internal standard solution, centrifuged, and the plasma supernatant isolated and stored in the dark. All of these steps were carried out immediately after receiving the blood. The DMTS concentration in the supernate was measured by HPLC following 0,3 , and 6 hours of storage.

The finalized sample preparation method for HPLC-UV detection of DMTS from blood was is summarized in Fig. 1. Specifically, $200 \mu \mathrm{l}$ of ice cold $0.1 \mathrm{mg} / \mathrm{ml}$ DMDS (internal standard) in acetonitrile and $80 \mu$ DMTS-exposed blood samples (originating either from spiking or in vivo experiments) were added to an Eppendorf tube. The Eppendorf tubes were vortexed for 10 minutes at room temperature, and then centrifuged at $14000 \mathrm{rcf}$ at $4^{\circ} \mathrm{C}$ for 5 minutes. Eighty microliters of the clear supernatants were transferred to HPLC vials with glass insert; from which, $40 \mu \mathrm{l}$ of sample was injected to the HPLC column. Isocratic 
elution was employed with a 35:65 v/v blend of water and acetonitrile flowing at a rate of 1 $\mathrm{ml} / \mathrm{min}$. To minimize delays, the flow of the experiment was contiguous.

\subsection{Method validation for DMTS detection from blood}

For the method validation, multiple samples of sheep blood were spiked with equal volumes of increasingly concentrated $(10-10000 \mu \mathrm{g} / \mathrm{ml})$ ethanolic DMTS solutions, to yield standard calibration solutions spanning a concentration range of $0.1-100 \mu \mathrm{g} / \mathrm{ml}$. The limits of detection (LOD), and quantitation (LOQ) were calculated to be, respectively, 3.3x and 10x the quotient obtained by dividing the residual standard deviation from the regression line, by the regression line slope [15]. Quality control (QC) samples with low, medium, and high DMTS concentrations were used to measure the precision and accuracy of the method. The relative standard deviation $(\mathrm{CV} \%)$ was employed as the figure of merit for precision. Accuracy was calculated as the ratio of the mean measured concentration to the theoretical value and expressed as a relative percentage. Intra and inter-day precision and accuracy were calculated based on five replicate measurements of the QC standards repeated on two different days. The recovery of DMTS from blood was calculated as the ratio of the slopes of the calibration curves for DMTS in blood and in ethanol, and then expressed as a percentage.

\subsection{Animals used in studies of DMTS detection from brain}

Male CD-1 mice (Charles River Laboratories, Inc., Wilmington, MA, USA) weighing 18-28 g were housed at $23^{\circ} \mathrm{C}$ in a light-controlled room (12-hour light/dark, full-spectrum lighting cycle with no twilight), and were furnished with water and $4 \%$ Rodent Chow (Harlan Laboratories Inc., Indianapolis, IN, USA) ad libitum. All animal procedures were conducted in accordance with the guidelines in The Guide for the Care and Use of Laboratory Animals [16], in a facility accredited by AAALAC (Association for Assessment and Accreditation of Laboratory Animal Care, International). Animals were euthanized in accordance with the AVMA Guidelines for the Euthanasia of Animals: 2013 Edition [17]. After euthanization the heads of the animals were removed and the brains were collected for analytical method development and validation for brain. The Institutional Animal Care and Use Committee (IACUC) permission number is 15-09-14-1015-3-01.

\subsection{Method development for DMTS detection from brain}

A GC-MS method was developed for the detection of DMTS from brain tissue. Mouse brains were divided into two halves. Individual brain halves were weighed, mixed with $1 \mathrm{ml}$ 
solvent $(15 \% \mathrm{w} / \mathrm{w}$ aq. polysorbate 80 , or ethanol) for each $220 \mathrm{mg}$ brain, transferred to vials containing $1.4 \mathrm{~mm}$ diameter ceramic beads. Each vial was homogenized three times at $6500 \mathrm{rpm}$ for 1 minute with a Precellys 24 tissue homogenizer (Bertin Technologies, Montigny-le-Bretonneux, France).

A $475 \mu 1$ aliquot of brain homogenate was transferred into a headspace sampling vial, spiked with internal standard ( $25 \mu 1$ of $1 \mathrm{mg} / \mathrm{ml}$ ethanolic dibutyl disulfide (DBDS)), sealed, vortexed for five seconds, and allowed to stand for five minutes. A solid phase micro extraction (SPME) fiber (100 $\mu \mathrm{m}$ polydimethyl siloxane coating) was inserted through a septum into the headspace above the spiked brain homogenate, and allowed to equilibrate for 10 minutes. The effects of stirring during the fiber incubation, and of adding sodium chloride (20 $\mathrm{w} / \mathrm{v} \%$ final concentration) to the spiked homogenate were tested. For analytical calculations, a brain density $0.883 \mathrm{mg} / \mu \mathrm{L}$ was used based upon a mouse whole brain mass of $400 \mathrm{mg}$ and volume of $453 \mu \mathrm{L}[18,19]$. This enabled the brain sample volume, $\mathrm{V}_{\mathrm{B}}$, and brain concentration, $\mathrm{C}_{\mathrm{B}}$, per milliliter of homogenate to be estimated using equations 1 and 2 .

$$
\begin{array}{ll}
V_{B}=220 m g_{B}\left(\frac{\mu L}{0.883 m g}\right)\left(\frac{m L}{1000 \mu L}\right)=0.249 m L & \text { Equation 1 } \\
C_{B}=\frac{220 m g_{\text {Brain }}}{0.249 m L_{\text {Brain }}+1 m L_{\text {sovvent }}}=176 \frac{m g_{B}}{m L_{\text {homogenate }}} & \text { Equation 2 }
\end{array}
$$

The brain sample gathered by SPME was analyzed with an Agilent Model 6890 gas chromatograph (Agilent DB-5MS column: $30 \mathrm{~m} \times 0.25 \mathrm{~mm}$ with $0.1 \mu \mathrm{m}$ thick stationary phase) coupled to an Agilent Model 5973 quadrupole mass spectrometer. Helium flowing at a rate of $1 \mathrm{ml} /$ minute was used as the mobile phase. The inlet temperature was $250{ }^{\circ} \mathrm{C}$. The column temperature was held at $40{ }^{\circ} \mathrm{C}$ for 1 minute, increased to $280{ }^{\circ} \mathrm{C}$ using a $60{ }^{\circ} \mathrm{C} /$ minute ramp, and then held at $280{ }^{\circ} \mathrm{C}$ for 3 minutes. The source and quadrupole temperatures were set at 230 and $150^{\circ} \mathrm{C}$, respectively. In the exploratory scan mode experiments, the $\mathrm{m} / \mathrm{z}$ ratio was scanned from 30 to 200. In the single ion monitoring (SIM) experiments used for quantitation, DMTS was quantified using ions at $\mathrm{m} / \mathrm{z}$ ratios of 44.9, 45.0, 63.9, 64.0, 78.9, $79.0,110.8,111.0,125.9,126.0$, and DBDS was quantified using ions at $\mathrm{m} / \mathrm{z}$ ratios of 178 and 178.1. Agilent ChemStation Version E.02.02.1431 software was used to process the data.

The finalized sample preparation method for GC-MS detection of DMTS from brain is summarized in Fig. 2. Specifically, half mouse brains were weighed and $1 \mathrm{ml}$ ethanol was added to $220 \mathrm{mg}$ brain. Brains were homogenized as previously described and $588 \mu \mathrm{l}$ of homogenates were spiked with $12 \mu \mathrm{l}$ of various concentration of DMTS/ethanol solutions. 
$475 \mu 1$ of the spiked homogenized brain was added to $25 \mu 1$ of $1 \mathrm{mg} / \mathrm{ml}$ DBDS (in ethanol) solution. A magnet bar was placed into the GC-MS vials and the samples were stirred for 5 minutes. The PDMS fiber was put into the headspace of the vials and incubated for 10 minutes, then the GC-MS was used to detect the molecules from the fiber.

\subsection{Method validation for DMTS detection from brain}

For method validation, multiple samples of homogenized brains or ethanol were spiked with an equal volume of increasingly concentrated $(0.15-50 \mu \mathrm{g} / \mathrm{ml})$ ethanolic DMTS solution, to yield standard calibration solutions spanning a concentration range of 54$5435 \mathrm{ng}_{\text {DMTS }} / \mathrm{g}_{\text {brain }}$. Linearity, limit of detection, and limit of quantification were calculated. Linearity was evaluated with using linear regression analysis. The limits of detection (LOD), and quantitation (LOQ) were calculated to be, respectively, 3.3x and 10x the quotient obtained by dividing the residual standard deviation from the regression line, by the regression line slope [15]. Quality control (QC) samples with low, medium, and high DMTS concentrations were used to measure the precision and accuracy of the assay method. The precision of the analytical method was estimated using the relative standard deviation $(\mathrm{CV} \%)$. Accuracy was defined as the ratio of the mean observed value to the theoretical value and was expressed as a relative percentage. Intra and inter-day precision and accuracy were calculated based on five replicate measurements of the QC standards taken on two different days. Recovery of DMTS from brain was calculated as the ratio of the slopes of the calibration curves for DMTS in brain and in ethanol, and then expressed as a percentage.

\subsection{Statistical analysis}

All plotted values represent means and all plotted error bars represent standard deviations (SD). A two-way analysis of variance (ANOVA) followed by a Bonferroni posttest (GraphPad Prism 5.0, GraphPad Software Inc., San Diego, CA, USA) was employed to compare the effect of organic solvents on the recovery of DMTS from spiked blood and brain samples. A one-way ANOVA followed by Dunnett's test was used to evaluate the effects of sonication, heating, and $\mathrm{NaOH}$ on DMTS recoveries from blood. Unpaired t-tests were utilized to test the significance of changes in signals associated with SIM and blood age. If the random probability ( $p$ ) of the observed change in signal associated with a specific treatment was less than 0.05 , then the effect of the variable under study was considered statistically significant. The number of replicate samples varied between 3 and 10 . 


\section{Results and Discussion}

\subsection{Method development for DMTS detection from blood}

Different sample preparation methods, injection volumes, and mobile phase blends were tested for possible improvements to the quality of the HPLC signal for DMTS detected from blood. In preliminary studies acetonitrile-water mobile phase blends were found to give more symmetric DMTS peak shapes than methanol-ethanol blends (unpublished data). To find a suitable mobile phase blend ratio, acetonitrile-water ratios of 60:40, 65:35, and 70:30 were tested (Fig. 3A). The 60:40 ratio resulted in longer retention times, and the more rapidly eluting 70:30 ratio resulted in poorer resolution of the DMTS peak from its neighbors. The 65:35 acetonitrile-water blend was selected as a good compromise between resolution, and speed of analysis. Of the three different HPLC injection volumes tested $(20,30,40 \mu 1)$, the 40 $\mu \mathrm{l}$ injections maintained good peak shapes while also yielding the highest DMTS and DMDS peak areas (Fig. 3B). Thus, in subsequent experiments $40 \mu$ l injection volumes were employed.

As part of the sample preparation protocol, organic solvents were added to the DMTS spiked blood to lyse the cells, and to precipitate protein aggregates and dissolve cell membranes (Fig. 4A). Methanol, ethanol, and acetonitrile were tested as solvent. The ethanol and acetonitrile addition yielded similar DMTS recoveries that were higher than those obtained from methanol. Acetonitrile was selected as the solvent for future experiments because it yielded precipitates that were less easily re-suspended than those obtained with the alcohols. This observation is consistent with other reports stating that acetonitrile has a stronger ability to precipitate proteins than ethanol or methanol [20]. Chilling the acetonitrile to $-20^{\circ} \mathrm{C}$ significantly increased the DMTS peak area at $100 \mu \mathrm{g} / \mathrm{ml}$ concentration, and decreased the standard deviation (Fig. 4B). Although it was hypothesized that sonication and heating might facilitate the release of DMTS from cells and/or proteins, both were found to reduce DMTS recovery (Fig. 4C).

\subsection{Validation the method of DMTS detection from blood}

Representative chromatograms for analytical samples prepared from raw sheep blood samples spiked respectively with 0,1 and $30 \mu \mathrm{g} / \mathrm{ml}$ of DMTS are presented in Fig. 5A. Additional standard calibration solutions were prepared in the range from 5 to $50 \mu \mathrm{g} / \mathrm{ml}$ by spiking sheep blood with appropriate volumes of ethanolic DMTS solution. The scatter plot 
of DMTS/DBDS peak area ratios versus the DMTS concentration exhibited good linearity (Fig. 5B). The equation for the regression was $\mathrm{y}=0.01161 \mathrm{x}-0.0091$, with the $\mathrm{R}^{2}=0.999$. The limit of detection and limit of quantification were calculated to be 1.46 and $4.45 \mu \mathrm{g} / \mathrm{ml}$, respectively. Because DMTS has excellent solubility in ethanol [7], a reference calibration curve was prepared based on standard solutions of DMTS in ethanol (Fig S1). The equation for DMTS calibration curve in ethanol was $\mathrm{y}=0.01979 \mathrm{x}-0.002$, and the related $\mathrm{R}^{2}$ was 0.999. By dividing the slopes of the calibration curves for DMTS from blood and ethanol, the relative recovery of DMTS from blood vs ethanol was calculated to be $58 \%$.

The intra- and inter-day precisions, and intra- and inter-day accuracies were estimated using low $(5 \mu \mathrm{g} / \mathrm{ml})$, medium $(30 \mu \mathrm{g} / \mathrm{ml})$, and high $(50 \mu \mathrm{g} / \mathrm{ml})$ concentrations of DMTS in sheep blood (Table 2). The intra- and inter-day precisions varied from 6.9 to $12.2 \mathrm{CV} \%$, while the intra- and inter-day accuracies varied from -3.7 to $-14.8 \%$. The FDA guidelines recommend that both precision and accuracy fall within a range of $\pm 15 \%$ [21].

Surprisingly, all of the mean concentrations measured in these intra- and inter-day validation experiments were lower than the nominal concentrations (Table 2). The same analyst, instrument, blood, DMTS solutions, and sample preparation methods were used in both the calibration curve and method validation experiments. However, because the calibration curve measurement was carried out 3-4 days after the method validation experiments, the blood and the DMTS solutions were both three days older. Because recent studies for DMTS solutions stored in sealed ampules showed excellent stability for 100 days (Petrikovics et al., unpublished data), changes in the DMTS stock solutions over the three days between experiments were ruled out as a cause of the systematic drop in observed signal. This led to a hypothesis that DMTS might be undergoing reactions in blood, and that these reactions might proceed more rapidly in fresh than in aged blood. To test this hypothesis, signals from sheep blood that was spiked with DMTS and analyzed immediately after receipt from the Carolina Company (0-day old blood), were compared to signals from sheep blood that was stored for three days (3-day old blood) prior to being spiked with DMTS and analyzed (Fig. 6A). DMTS recovery was significantly higher from the 3-day old blood. Based on these results, we conclude that a) DMTS interacts with blood in a manner that lowers the DMTS recovery, b) this interaction is more pronounced in fresh blood than in older blood. Investigation of the interaction between DMTS and blood was not the aim of this study; however, this observed interaction has prompted further studies, which are ongoing, to 
determine the detailed mechanism of this interaction, and to determine whether it is also present in human blood. These results will be published later.

The observation that DMTS concentrations decrease with time in blood, and that this decrease is more pronounced in fresh blood, places important constraints on the method. Specifically, it is crucial that blood samples be stabilized immediately after collection. Toward this end, solutions were prepared by mixing DMTS-spiked blood with acetonitrile and then immediately centrifuging to remove precipitated proteins (Fig. 6B). These solutions were analyzed for DMTS following incubation periods of 0,3 , and 6 hours. No statistically significant variation in DMTS concentration were observed from the variously incubated mixtures (Fig. 6B). Thus, mixing blood samples with acetonitrile immediately upon collection, centrifuging and isolating the supernatant provides a method of stabilizing samples for subsequent analysis. The preservative effects of acetonitrile are likely due to protein denaturing that terminates enzymatic activities, and that facilitates protein removal by centrifugal sedimentation.

Because DMTS holds considerable promise as a cyanide antidote, this method for stabilizing and then detecting DMTS from blood provides an important tool for further studies.

\subsection{Method development for DMTS detection from brain}

A method was developed for detecting DMTS from mouse brain. The isolated mouse brains were spiked with DMTS and different sample preparations were applied to gain the highest sensitivity for the DMTS detection. The following conditions were tested (Fig. 7): I) brain homogenization in $15 \%(\mathrm{w} / \mathrm{w})$ aq. polysorbate 80 and ethanol; II) applying stirring during DMTS extraction; III) adding salt to enhance the DMTS evaporation.

Ethanol and $15 \%(\mathrm{w} / \mathrm{w})$ aq. polysorbate 80 , were all tested as possible solvents for the homogenization of the brain samples (Fig. 7A). Ethanol yielded the highest DMTS recoveries, and was thus selected as the solvent for subsequent experiments. Stirring the brain homogenate significantly increased the peak areas of DMTS and decreased the standard deviations of the peak (Fig. 7B). Adding salt did not have a significant effect on DMTS peak area (Fig. 7C). Based on these results an improved method was developed for DMTS detection from mouse brain (Fig. 2).

\subsection{Validation the method of DMTS detection from brain}


A representative mass spectrum for DMTS is presented in Fig. 8A. Representative chromatograms from brain spiked with 0,326 and $5435 \mathrm{ng}$ of DMTS per gram of brain are shown in Fig. 8B. Calibration curves (plotting DMTS:DBDS peak area ratios versus DMTS concentration), were prepared for DMTS spiked brain (Fig. 9), and ethanol (Fig S1B) solutions. The linearity of the brain calibration curve was determined between 54 and 5435 ng DMTS/gram brain, and the coefficient of the determination $\left(\mathrm{R}^{2}\right)$ was 0.995 . The calibration equation for DMTS in brain was $\mathrm{y}=3.765 \cdot 10^{-6} \mathrm{x}+3.312 \cdot 10^{-4}$, when the units for $\mathrm{x}$ were $\mathrm{ng}_{\text {DMTS }} / \mathrm{g}_{\text {brain }}$, and $\mathrm{y}=2.046 \cdot 10^{-5} \mathrm{x}+3.312 \cdot 10^{-4}$ when the units for $\mathrm{x}$ were

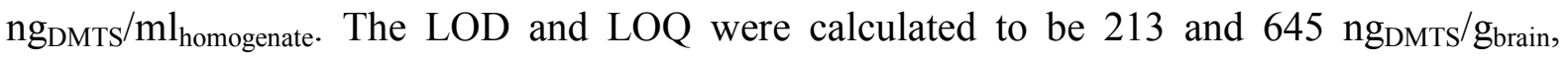
respectively. The same detection method was applied for DMTS analysis from ethanol solution (Fig. S1B). The calibration equation and coefficient of the determination for DMTS in ethanol were, respectively, $\mathrm{y}=5.811 \cdot 10^{-5} \mathrm{x}-4 \cdot 10^{-4}$, and and $\mathrm{R}^{2}=0.996$, when the units for $\mathrm{x}$ were $\mathrm{ng}_{\mathrm{DMTS}} / \mathrm{ml}$. Recovery of DMTS from brain was calculated by taking the ratio of the slopes of the calibration curves for DMTS in brain and in ethanol, and was found to be $35 \%$.

Intra- and inter-day precision and accuracy were determined in mouse brain homogenate for DMTS using quality control solutions having low (326), medium (1630), and high $(5435 \mathrm{ng} / \mathrm{g})$ DMTS concentrations in brain (Table 3). The precisions were below 24.3 CV\% while the accuracy was between -1.3 and $+2.4 \%$. According to the FDA guideline, the precision and the accuracy need to be below $\pm 15 \%$ [21]. In the ongoing quest to better understand $\mathrm{CN}$ intoxication and antagonism, where in vivo samples are limited, and study of antidotal biochemistry is ongoing, this method represents an important step forward for pharmacokinetics and detoxification studies of the brain in the presence of cyanide and DMTS.

\section{Conclusion}

DMTS is a promising cyanide antagonist [7,9]. Investigation of DMTS requires good analytical methods for biological samples. In this study analytical methods were developed for DMTS detection from blood by HPLC and from brain by GC-MS. Different sample preparations and instrumental settings were investigated to improve the quality and sensitivity of detection. The DMTS analytical method for blood presented here complements the stir bar sorptive extraction GCMS method employed by Manandhar et al. [14]. The DMTS analytical method for brain presented here, is to the best of our knowledge, the first reported. 
While working to validate the method for detecting DMTS from blood, a new phenomenon was observed. The DMTS recovery from fresh blood was significantly lower than from aged blood. These low recoveries of DMTS from fresh blood are hypothesized to be due to reaction of the DMTS with blood components. These reactions may represent a loss mechanism for the antidote, or may be part of the mechanism by which DMTS detoxifies cyanide in the blood, and thus present an important avenue for future studies. Rapid extraction of the plasma from DMTS exposed blood samples was found to effectively stabilize the DMTS concentrations over a period of 6 hours, and thus provides a method for overcoming the inter-day accuracy challenge that was encountered in the analytical method for determining DMTS from blood. In the brain method the precision was lower than that recommended by the FDA [21]. Precision will be improved in the future by taking volume and density measurements of brain homogenate in order to reduce the number of assumptions built into the model for calculating DMTS concentrations in the homogenate. The analytical methods presented here represent important steps forward for the in vivo determination of DMTS concentrations in blood and brain and provide a strong foundation for further pharmacokinetics investigations.

\section{Acknowledgement}

This research was supported by the CounterACT Program, National Institutes of Health Office of the Director, and the National Institute of Allergy and Infectious Diseases, NIH/ Department of Defense Interagency Agreement AOD12060-001-00000/A120-B.P2012-01), and the Robert A. Welch Foundation (X-0011) at Sam Houston State University, Huntsville, TX.

\section{Conflict of interest}

The authors report no conflicts of interest. The authors alone are responsible for the content and writing of the paper.

\section{Disclosure}

Patent, related to DMTS: Ilona Petrikovics (SHSU), Steven Baskin (ICD), and Gary Rockwood (ICD). Dimethyltrisulfide as a cyanide antidote (2015 October), Patent No.\#: US20150290143 A1. 


\section{Figure legends}

Fig. 1 Detection method for DMTS analysis from blood by HPLC. DMTS, dimethyl trisulfide, is the analyte; DMDS, dimethyl disulfide, is the internal standard.

Fig. 2 Method for DMTS analysis from brain by GC-MS. DMTS, dimethyl trisulfide, is the analyte; DBDS, dibutyl disulfide, is the internal standard; EtOH, ethanol; SPME, solid phase microextraction; PDMS, polydimethylsiloxane.

Fig. 3 Improving the method for DMTS detection from blood by investigating the effect of mobile phase and injection volume. (A) Appling different acetonitrile-water ratios to get better peaks for DMTS and DMDS. (B) Applying increasing injection volumes to result higher DMTS and DMDS peaks. DMTS, dimethyl trisulfide, is the analyte; DMDS, dimethyl disulfide, is the internal standard.

Fig. 4 Improving the method for DMTS detection from blood by investigating the effect of different sample preparation techniques. (A) DMTS spiked blood samples $(10$ and $100 \mu \mathrm{g} / \mathrm{ml}$ of DMTS) were exposed to ethanol, acetonitrile, and methanol and the effects of these solvents on DMTS peak were investigated. (B) DMTS spiked blood samples (10 and 100 $\mu \mathrm{g} / \mathrm{ml}$ of DMTS) were exposed to room temperature acetonitrile, and ice cold acetonitrile and the effects of these solvents on DMTS peak were investigated. (C) Blood samples spiked with DMTS (final concentration: $500 \mu \mathrm{g} / \mathrm{ml}$ ) were treated with sonication, and heat $\left(100^{\circ} \mathrm{C}\right)$ and the effects of these on DMTS peak were followed. Data are presented as mean \pm S.D., $n$ = 3; statistical analysis: two-way ANOVA followed by Bonferroni test (A, B) or ANOVA followed by Dunnett's test (C). Effects of organic solvents (A): statistically significant difference was detected after treatment with ethanol $(* \mathrm{P}<0.05)$ compared to the methanol group at $100 \mu \mathrm{g} / \mathrm{ml}$. Effects of the temperature of the acetonitrile (B): statistically significant difference was detected after treatment with cold ACN $(* \mathrm{P}<0.05)$ compared to the ACN group at $100 \mu \mathrm{g} / \mathrm{ml}$. Effects of sonication and heat $(\mathrm{C})$ : treatment groups were compared to their respective control $(* * * \mathrm{P}<0.001)$. DMTS, dimethyl trisulfide, is the analyte; DMDS, dimethyl disulfide, is the internal standard. Abbreviations: EtOH, ethanol; ACN, acetonitrile; Cold ACN, ice cold acetonitrile; $\mathrm{MeOH}$, methanol; Sonic., sonication; conc., concentration.

Fig. 5 Chromatograms and calibration curves for DMTS detection from sheep blood. (A) Representative chromatograms for DMTS and DMDS detection from sheep blood. (B) Calibration curve for DMTS detection from spiked sheep blood. DMTS, dimethyl trisulfide, is the analyte; DMDS, dimethyl disulfide, is the internal standard.

Fig. 6 Effect of blood age on the DMTS peak and DMTS incubation in acetonitrile treated blood. (A) Fresh and 3-day old defibrinated sheep blood was spiked with DMTS and compared. (B) DMTS spiked blood samples were added to acetonitrile-DMDS mixture, then precipitates were removed by centrifugation and the supernatants were incubated for 0,3 and 6 hours. Data are presented as mean \pm S.D., $n=4-7$; statistical analysis: unpaired t-test for (A) and ANOVA followed by Dunnett test for (B). Statistically significant difference was detected after comparing the two blood conditions $(* \mathrm{P}<0.05)$. 
Fig. 7 Improving the method for DMTS detection from mouse brain by investigating the effect of different sample preparation and detection techniques. (A) Whole brains were homogenized in $15 \mathrm{w} / \mathrm{w} \%$ aq. polysorbate 80 or ethanol, then spiked with DMTS and the effects of these solvents/solutions on DMTS peak were analyzed. (B) DMTS spiked homogenized brain samples were or were not stirred during head space sampling and the effect of stirring on DMTS peaks was analyzed. (C) Salt (sodium chloride) was added to homogenized brains spiked with DMTS and the effect of $\mathrm{NaCl}$ addition on DMTS peak was analyzed. Data are presented as mean \pm S.D., $n=3$; statistical analysis: Student t-test. Effects of solvents on the homogenization (A): statistically significant differences were detected after treatments with ethanol $(* * \mathrm{P}<0.01)$ in DMTS analysis compared to the $15 \mathrm{w} / \mathrm{w} \%$ aq. polysorbate 80 groups. Effects of stirring (B): statistically significant difference was detected after stirring $(* \mathrm{P}<0.05)$ compared to the non-stirring group at DMTS analysis. Effect of salt (C): there was no statistically significant difference. DMTS, dimethyl trisulfide, is the analyte. Abbreviations: EtOH, ethanol; Poly $80,15 \mathrm{w} / \mathrm{w} \%$ aq. polysorbate 80 .

Fig. 8 Ion in peak of DMTS molecule and GC-MS chromatograms when DMTS was detected from brain. (A) Ions related for DMTS and their abundance. (B) Representative chromatograms for DMTS and DBDS detection from mouse brain. DMTS, dimethyl trisulfide, is the analyte; DBDS, dibutyl disulfide, is the internal standard.

Fig. 9 Calibration curve for DMTS detection from mouse brain. Peak area ratio of DMTS/DBDS versus ng DMTS/gram brain was plotted. DMTS, dimethyl trisulfide, is the analyte; DBDS, dibutyl disulfide, is the internal standard.

Fig. S1 Calibration curves for DMTS detection from ethanol by HPLC and GC-MS. (A) DMTS was detected from ethanol solvent by HPLC using the method developed for DMTS analysis from blood. (B) DMTS was detected from ethanol solvent by GC-MS/SPME using the method developed for DMTS analysis from brain. Peak area ratio of DMTS/DMDS or DMTS/DBDS versus DMTS nominal concentration were plotted. DMTS, dimethyl trisulfide, is the analyte; DMDS, dimethyl disulfide, is the internal standard for HPLC measurement; DBDS, dibutyl disulfide, is the internal standard for GC-MS analysis. 
Table 1 Physicochemical properties of DMTS, DMDS and DBDS

\begin{tabular}{|c|c|c|c|}
\hline Name & $\begin{array}{c}\text { Dimethyl Trisulfide } \\
\text { (DMTS) }\end{array}$ & $\begin{array}{c}\text { Dimethyl Disulfide } \\
\text { (DMDS) }\end{array}$ & $\begin{array}{c}\text { Dibutyl Disulfide } \\
\text { (DBDS) }\end{array}$ \\
\hline \multicolumn{4}{|l|}{ Structure } \\
\hline Note & Antidote & $\begin{array}{l}\text { Internal standard for } \\
\text { DMTS in HPLC analysis }\end{array}$ & $\begin{array}{l}\text { Internal standard for DMTS in GC-MS } \\
\text { analysis }\end{array}$ \\
\hline CAS Number & $3658-80-8$ & $624-92-0$ & $629-45-8$ \\
\hline Boiling point & $165-170^{\circ} \mathrm{C}(760 \mathrm{mmHg})$ & $109-110^{\circ} \mathrm{C}(760 \mathrm{mmHg})^{3}$ & $226.0 \pm 0.0^{\circ} \mathrm{C}$ at $760 \mathrm{mmHg}^{5}$ \\
\hline $\log P$ & $2.93^{2}$ & $1.77^{4}$ & $4.96^{5}$ \\
\hline Vapour Pressure & $1.1 \pm 0.3 \mathrm{mmHg}$ at $25^{\circ} \mathrm{C}^{2}$ & $28.7 \pm 0.2 \mathrm{mmHg}$ at $25^{\circ} \mathrm{C}^{4}$ & $0.1 \pm 0.4 \mathrm{mmHg}$ at $25^{\circ} \mathrm{C}^{5}$ \\
\hline Water solubility & insoluble & & \\
\hline
\end{tabular}

${ }^{1}$ Food and Agriculture Organization of the United Nations (http://www.fao.org/ag/agn/jecfa-flav/details. html?flavld=2663)

${ }^{2}$ Predicted data is generated using the ACD/Labs Percepta Platform - PhysChem Module (http://www.chemspider.com/Chemical-Structure.18219.html)

${ }^{3}$ Food and Agriculture Organization of the United Nations (http://www.fao.org/ag/agn/jecfa-flav/details.html?flavld=4324)

${ }^{4}$ Predicted data is generated using the ACD/Labs Percepta Platform - PhysChem Module (http://www.chemspider.com/Chemical-Structure.11731.htm/?rid=c1ff3e0b-4b47-4d3e-89e4-323353dc5693)

${ }^{5}$ Predicted data is generated using the ACD/Labs Percepta Platform - PhysChem Module (http://www.chemspider.com/Chemical-Structure.11880.htm/?rid=13856362-7b62-4ce0-8939-aac092a4208e)

Table 2. Precision and accuracy for determining DMTS in blood

\begin{tabular}{|c|c|c|c|}
\hline $\begin{array}{c}\text { Nominal concentration } \\
(\boldsymbol{\mu g} / \mathbf{m l})\end{array}$ & $\begin{array}{c}\text { Measured }^{\mathbf{a}} \\
(\boldsymbol{\mu g} / \mathbf{m l})\end{array}$ & $\begin{array}{c}\text { Precision }^{\mathbf{b}} \\
(\mathbf{C V} \mathbf{0})\end{array}$ & $\begin{array}{c}\text {Accuracy }^{\mathbf{c}} \\
(\text { Bias, \%) }\end{array}$ \\
\hline Intra-day $(\mathbf{n}=\mathbf{5})$ & & & \\
\hline 5 & $4.7 \pm 0.6$ & 12.2 & -5.2 \\
\hline 30 & $26.3 \pm 1.8$ & 6.9 & -12.2 \\
\hline 50 & $48.2 \pm 4.4$ & 9.1 & -3.7 \\
\hline & & & \\
\hline Inter-day $\left(\mathbf{n}_{\text {day1 }}=\mathbf{5 ,} \mathbf{n}_{\text {day2 }}=\mathbf{5}\right)$ & & & -7.6 \\
\hline 5 & $4.6 \pm 0.4$ & 9.7 & -14.8 \\
\hline 30 & $25.5 \pm 1.9$ & 7.5 & -8.1 \\
\hline 50 & $45.9 \pm 4.4$ & 9.6 & \\
\hline
\end{tabular}

\footnotetext{
${ }^{a}$ Mean \pm standard deviation

${ }^{b}$ (Standard deviation/measured mean concentration) $x 100(\%)$

${ }^{c}$ (Measured mean concentration/theoretical concentration) $x 100-100(\%)$

CV\%: relative standard deviation
} 
Table 3. Precision and accuracy for determining DMTS in brain

\begin{tabular}{|c|c|c|c|}
\hline $\begin{array}{c}\text { Nominal concentration } \\
\text { (ng } \\
\left.\text { DMTS } / \mathbf{g}_{\text {brain }}\right)\end{array}$ & $\begin{array}{c}\text { Measured }^{\mathrm{a}} \\
(\mu \mathrm{g} / \mathrm{ml})\end{array}$ & $\begin{array}{c}\text { Precision }^{b} \\
(\mathrm{CV} \%)\end{array}$ & $\begin{array}{r}\text { Accuracy }^{\mathrm{c}} \\
(\text { Bias, \%) }\end{array}$ \\
\hline \multicolumn{4}{|l|}{ Intra-day $(n=5)$} \\
\hline 326 & $332.2 \pm 45.1$ & 13.6 & 1.9 \\
\hline 1630 & $16609.9 \pm 233.5$ & 14.5 & -1.3 \\
\hline 5435 & $5456.8 \pm 658.5$ & 12.1 & 0.4 \\
\hline \multicolumn{4}{|l|}{ Inter-day $\left(n_{\text {day } 1}=3, n_{\text {day } 2}=3\right)$} \\
\hline 326 & $333.9 \pm 81.1$ & 24.3 & 2.4 \\
\hline 1630 & $1661.9 \pm 95.3$ & 5.7 & 1.9 \\
\hline 5435 & $5544.1 \pm 337.3$ & 6.1 & 2.0 \\
\hline
\end{tabular}

\footnotetext{
${ }^{\text {a }}$ Mean \pm standard deviation

${ }^{\mathrm{b}}$ (Standard deviation/measured mean concentration) x $100(\%)$

c (Measured mean concentration/theoretical concentration) x $100-100(\%)$
} 


\section{References}

[1] S.K. Banerjee and S.K. Maulik, Effect of garlic on cardiovascular disorders: a review, Nutr. J., 1 (2002) 4.

[2] P. Fuchsmann, M.T. Stern, Y.A. Brügger, K. Breme, Olfactometry profiles and quantitation of volatile sulfur compounds of swiss tilsit cheeses, J. Agric. Food Chem., 63 (2015) 7511-7521.

[3] Kosher Certified Products, download date: September 30 2015, webpage: http://www.sigmaaldrich.com/chemistry/chemistry-products.html?TablePage=110357983

[4] S. Paczkowski, S. Nicke, H. Ziegenhagen, S. Schütz, Volatile emission of decomposing pig carcasses (Sus scrofa domesticus L.) as an indicator for the postmortem interval, J. Forensic Sci., 60 (2015) S130-S137.

[5] S. Elkahoui, N. Djébali, N. Yaich, S. Azaiez, M. Hammami, R. Essid, F. Limam, Antifungal activity of volatile compounds-producing Pseudomonas P2 strain against Rhizoctonia solani, World J. Microbiol. Biotechnol., 31 (2015) 175-185.

[6] X.C. Liu, Q. Liu, L. Zhou, Z.L. Liu, Evaluation of larvicidal activity of the essential oil of Allium macrostemon Bunge and its selected major constituent compounds against Aedes albopictus (Diptera: Culicidae), Parasit. Vectors, 7 (2014) 184.

[7] K. Kovacs, A.C. Duke, M. Shifflet, B. Winner, S. A. Lee, G.A. Rockwood, I. Petrikovics, Parenteral dosage form development and testing of dimethyl trisulfide, as an antidote candidate to combat cyanide intoxication, Pharm. Dev. Technol., 7450 (2016) 1-6.

[8] I. Petrikovics, S.I. Baskin, G.A. Rockwood, US Patent, 2015, US20150290143 A1 Retrieved from http://www.google.com/patents/US20150290143

[9] G.A. Rockwood, D.E. Thompson, I. Petrikovics, Dimethyl trisulfide: A novel cyanide countermeasure, Toxicol. Ind. Health, 2016, pii: 0748233715622713

[10] I. Petrikovics, M. Budai, K. Kovacs, D.E. Thompson, Past, present and future of cyanide antagonism research: From the early remedies to the current therapies, World J. Methodol., 5 (2015) 88-100.

[11] M. Shirasu, S. Nagai, R. Hayashi, A. Ochiai, K. Touhara, Dimethyl trisulfide as a characteristic odor associated with fungating cancer wounds, Biosci. Biotechnol. Biochem., 73 (2009) 2117-2120.

[12] A. Isogai, R. Kanda, Y. Hiraga, T. Nishimura, H. Iwata, N. Goto-Yamamoto, Screening and identification of precursor compounds of dimethyl trisulfide (DMTS) in Japanese sake, J. Agric. Food Chem., 57 (2009) 189-195.

[13] M. Nedjma and N. Hoffmann, Hydrogen Sulfide Reactivity with Thiols in the Presence of Copper(II) in Hydroalcoholic Solutions or Cognac Brandies: Formation of Symmetrical and Unsymmetrical Dialkyl Trisulfides, J. Agric. Food Chem., 44 (1996) 3935-3938.

[14] E. Manandhar, N. Maslamani, I. Petrikovics, G.A. Rockwood, B.A. Logue, Determination of dimethyl trisulfide in rabbit blood using stir bar sorptive extraction gas chromatography-mass spectrometry, J. Chromatogr. A., 1461 (2016) 10-17.

[15] ICH Topic Q 2 (R1) Validation of Analytical Procedures: Text and Methodology, ICH, download date November 1 2015, webpage:

http://www.ema.europa.eu/docs/en_GB/document_library/Scientific_guideline/2009/09/WC50 0002662.pdf

[16] The Guide for the Care and Use of Laboratory Animals (National Academic Press, 2011), download date: August 20 2015, webpage: http://grants.nih.gov/grants/olaw/guide-for-the-careand-use-of-laboratory-animals.pdf 
[17] AVMA Guidelines for the Euthanasia of Animals: 2013 Edition, download date: August 20 2015, webpage: https://www.avma.org/KB/Policies/Documents/euthanasia.pdf

[18] T. Kobayashi, Brain-to-body ratios and time of maturation of the mouse brain, Am. J. Physiol., 204 (1963) 343-346.

[19] Y. Ma, P.R. Hof, S.C. Grant, S.J. Blackband, R. Bennett, L. Slatest, M.D. Mcguigan, H. Benveniste, A three-dimensional digital atlas database of the adult C57BL/6J mouse brain by magnetic resonance microscopy, Neuroscience, 135 (2005) 1203-1215.

[20] P. Li and M. G. Bartlett, A review of sample preparation methods for quantitation of smallmolecule analytes in brain tissue by liquid chromatography tandem mass spectrometry (LCMS/MS), Anal. Methods, 6 (2014) 6183-6207.

[21] Guidance for Industry, Bioanalytical Method Validation, FDA, download date: November 1 2015 , webpage: http://www.fda.gov/downloads/Drugs/GuidanceComplianceRegulatoryInformation/Guidances/ ucm070107.pdf 
DMTS exposed blood

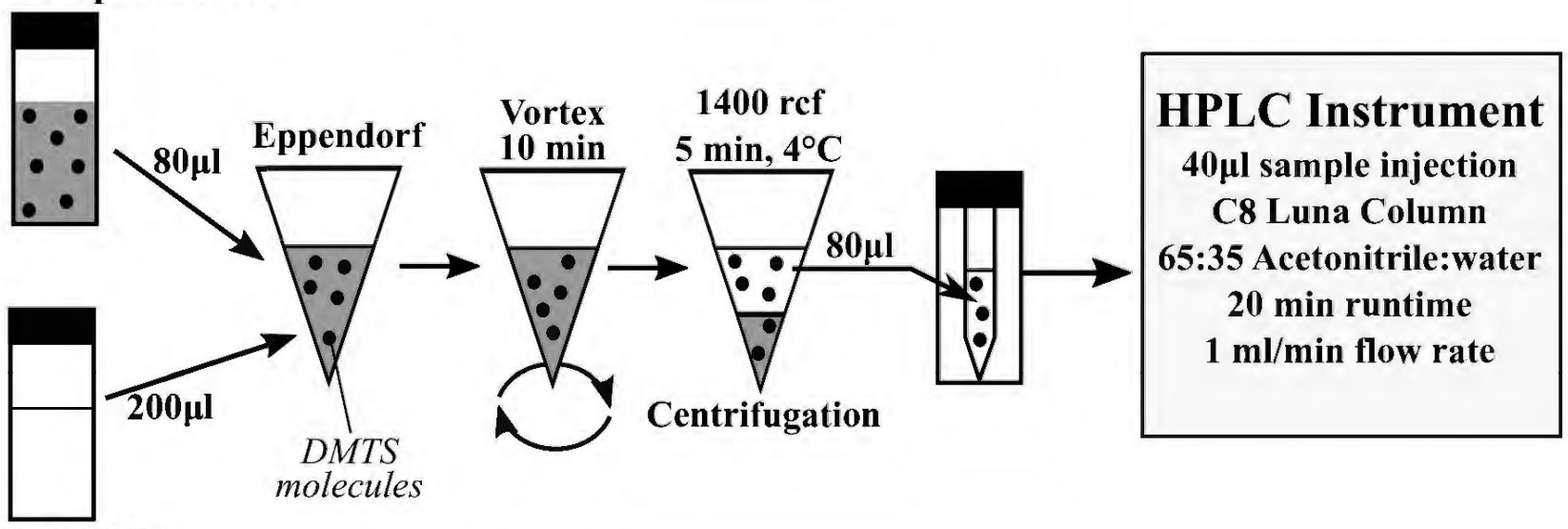

$0.1 \mathrm{mg} / \mathrm{ml}$ DMDS

in $\mathrm{ACN}$ 

A

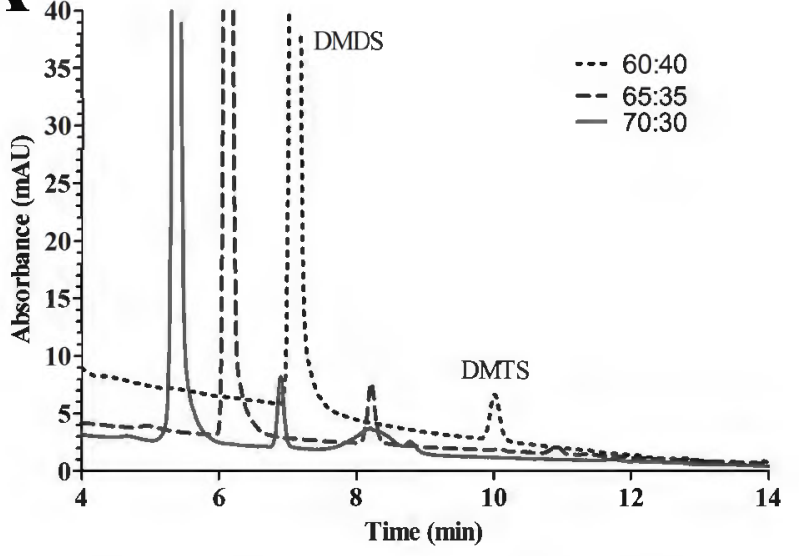

B $^{1500}$

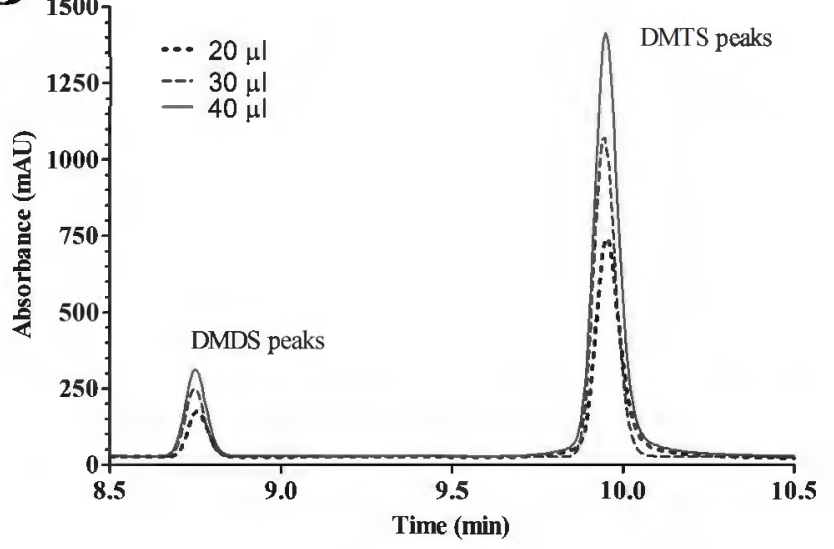



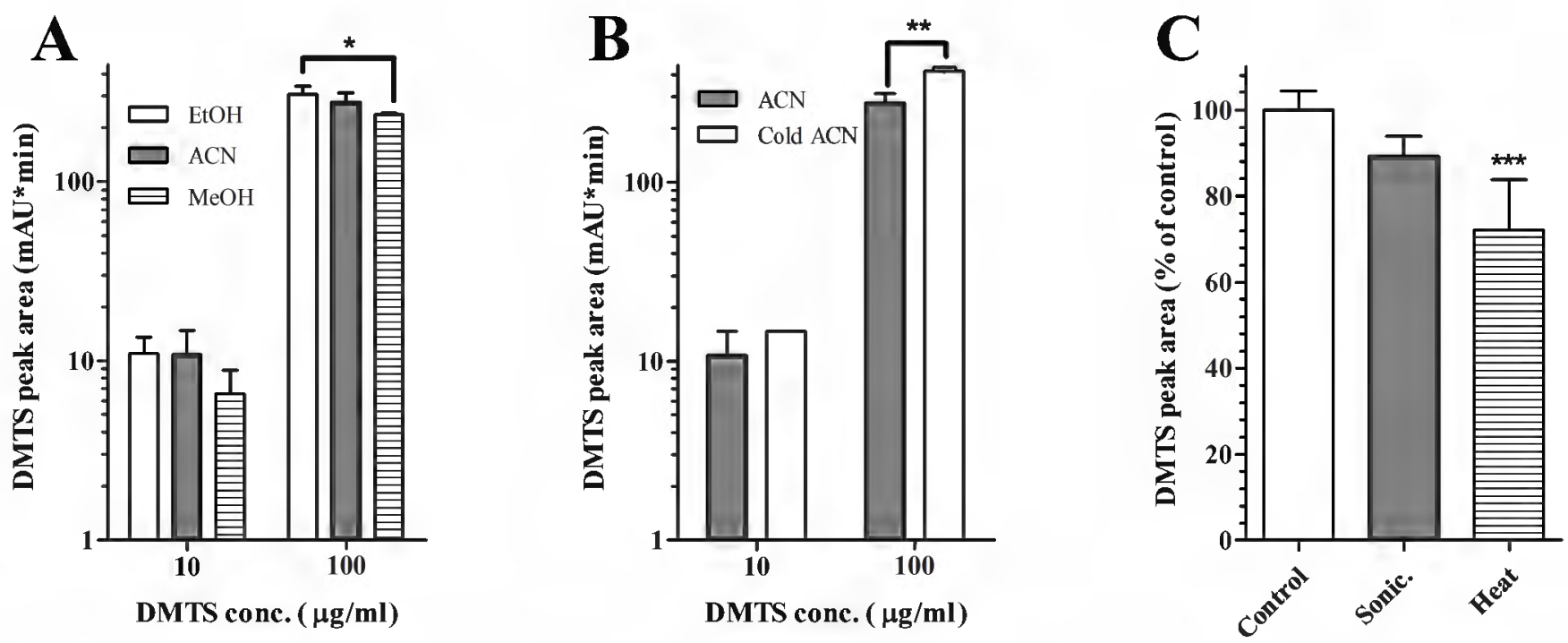

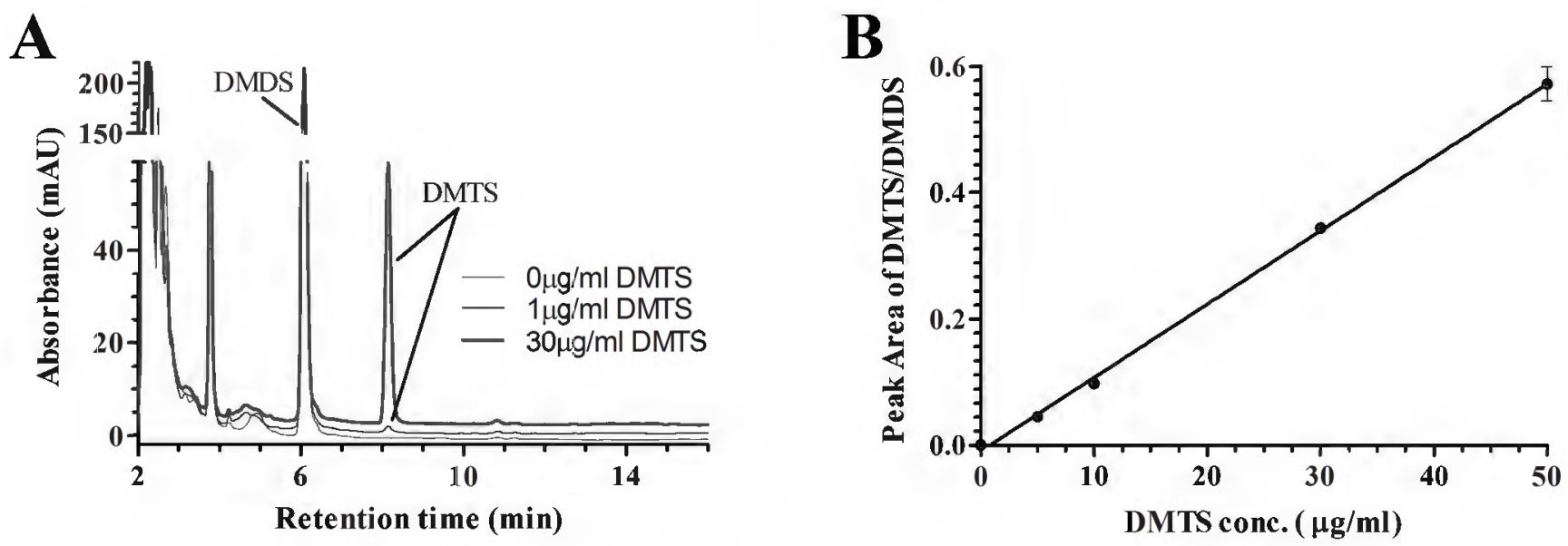


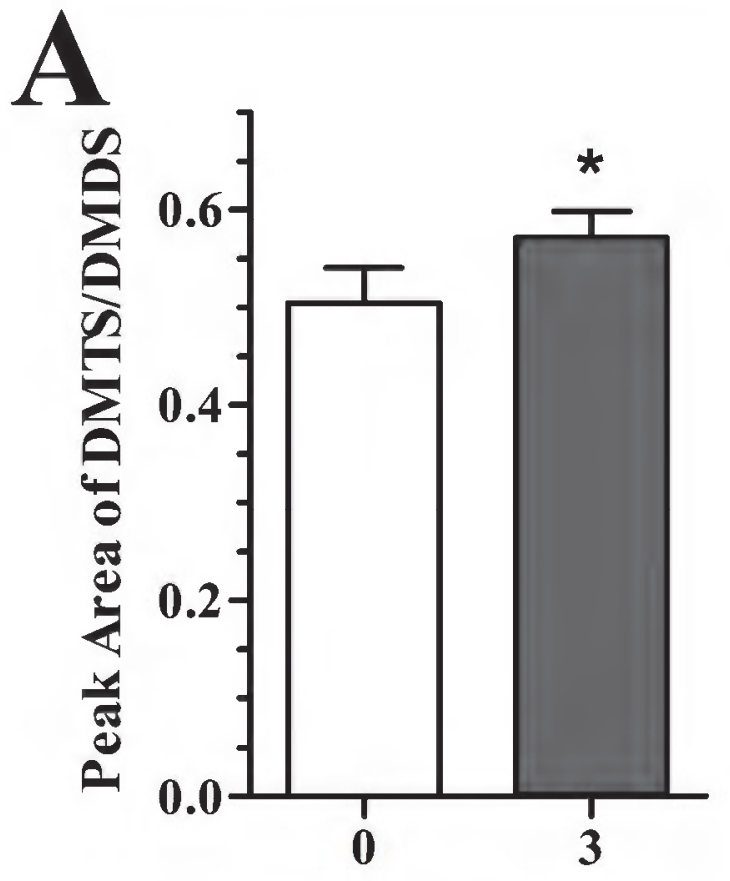

Blood age (days)
B

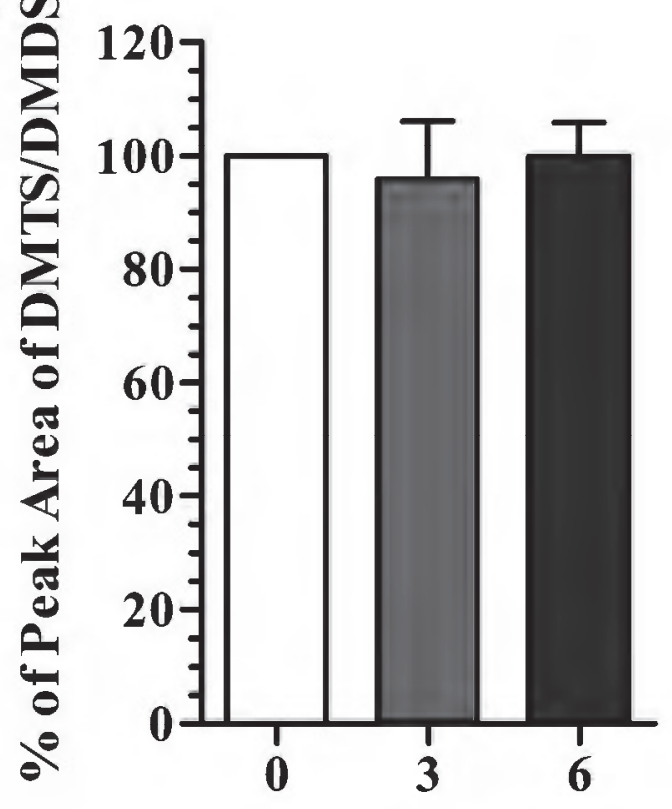

Incubation time (h) 
DMTS Peak area (ion abundace $x 10^{7}$ )

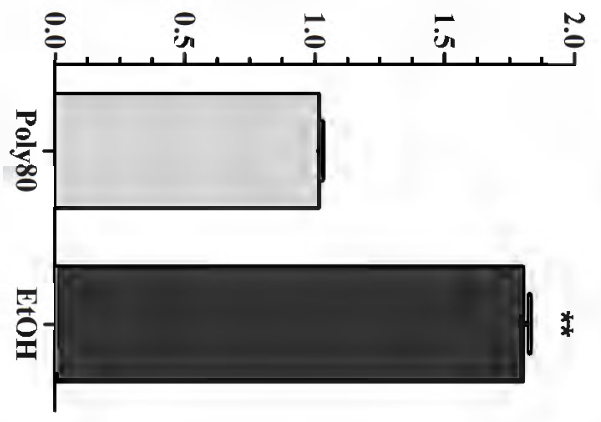

DMTS Peak area (ion abundace $x 10^{7}$ )

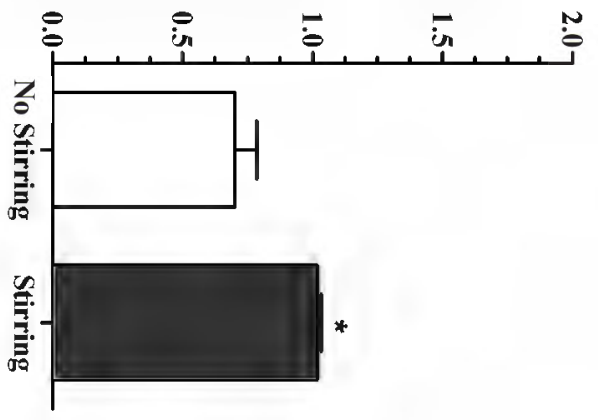

DMTS Peak area (ion abundace x 10

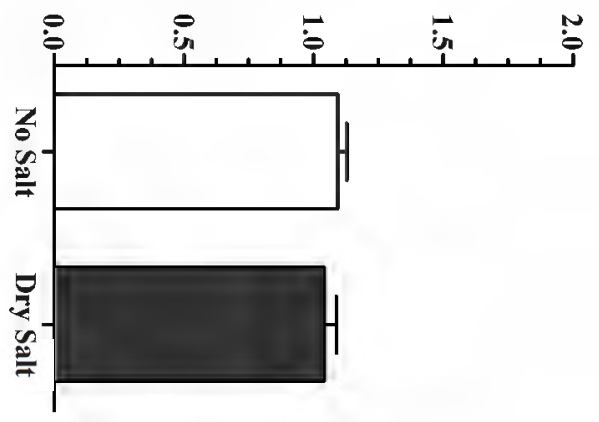



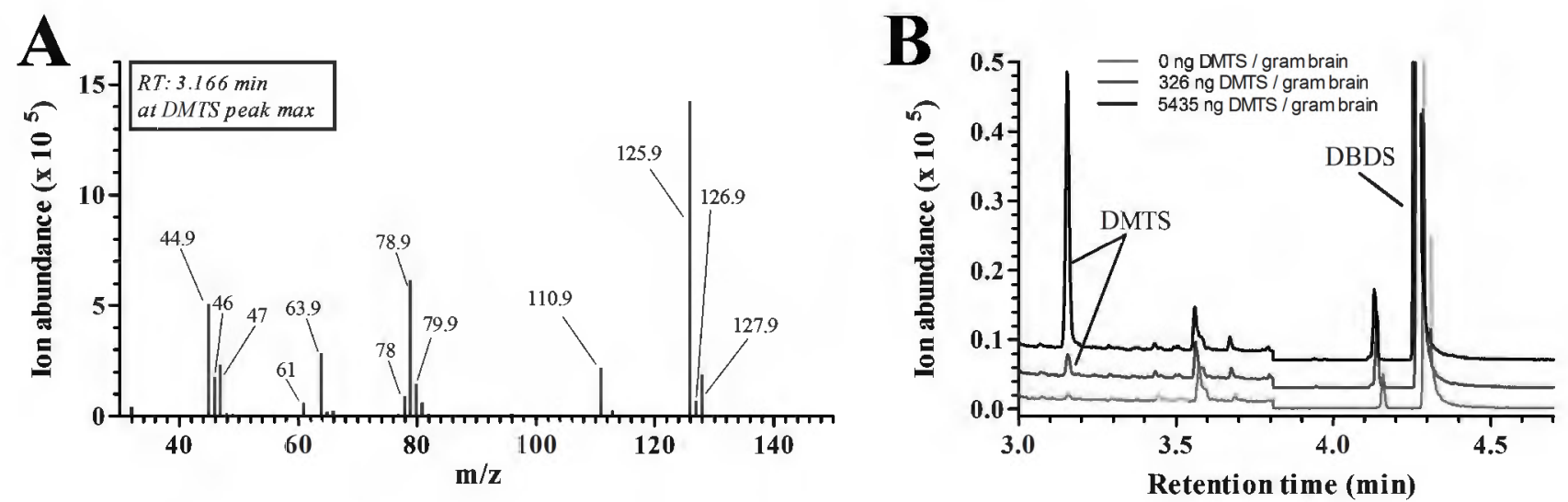


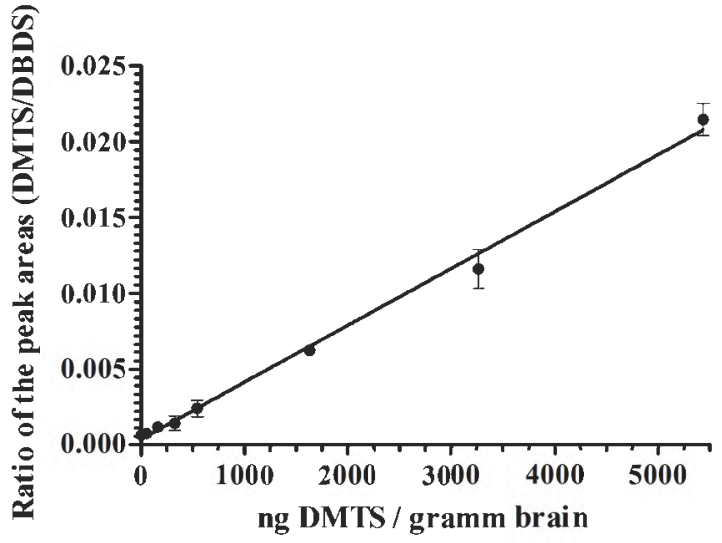

